\title{
Androgen Metabolism Process
}

National Cancer Institute

\section{Source}

National Cancer Institute. Androgen Metabolism Process. NCI Thesaurus. Code C19958.

Androgen Metabolism consists of diverse biochemical reactions in the adrenal cortex

that produce 19-carbon steroid hormones, which promote male sexual development or convert such steroids to metabolic derivatives. 